\title{
Postural Restriction in Benign Paroxysmal Positional Vertigo: A Randomized Clinical Trial
}

\author{
Yuvatiya Plodpai, M.D., Pattarawadee Prayuenyong, M.D.
}

Department of Otolaryngology, Faculty of Medicine, Prince of Songkla University, Hat Yai, Songkhla 90110, Thailand.

Received 29 May 2020 • Revised 10 July 2020 • Accepted 26 July 2020 • Published online 24 November 2020

\section{Abstract:}

Objective: We compared the efficacy between addition of post-maneuver postural restriction, and that of the modified Epley's maneuver alone.

Material and Methods: One hundred eighty patients with posterior canal benign paroxysmal positional vertigo were divided into two groups, using a block of four randomization. Group A were instructed to avoid head movement for 48 hours after the modified Epley's maneuver. Group B were treated with the modified Epley's maneuver alone. DixHallpike test and dizziness handicap inventory $(\mathrm{DHI})$ scores were assessed at 1 and 2 weeks, and followed up for 48 weeks to assess recurrent symptoms.

Results: There were no significant differences between the two groups in terms of changes from a positive to a negative Dix-Hallpike test; while the DHI score and recurrence rate were significantly lower in group A (p-value 0.042). Conclusion: Postural restriction after the modified Epley's maneuver can improve the DHI score, and reduce the recurrence rate of vertigo, compared with the modified Epley's maneuver alone.

Keywords: BPPV, posterior semicircular canal, postural restriction, vertigo

Contact: Assoc. Prof. Yuvatiya Plodpai, M.D.

Department of Otolaryngology, Faculty of Medicine,

Prince of Songkla University, Hat Yai, Songkhla 90110, Thailand.

E-mail: yuva078@hotmail.com

This is an open access article under the CC BY-NC-ND license

(http://www.jhsmr.org/index.php/jhsmr/about/editorialPolicies\#openAccessPolicy).

J Health Sci Med Res 2021;39(3):201-206 doi: 10.31584 /jhsmr.2020776 www.jhsmr.org 


\section{Introduction}

Benign paroxysmal positional vertigo (BPPV) is the most common disorder of the vestibular system. ${ }^{1}$ Von Brevern and colleagues reported a 1-year prevalence of $1.6 \%$ and a lifetime prevalence of $2.4 \% .^{2}$ The typical clinical manifestation is acute episodic vertigo, which lasts $<1 \mathrm{~min}$ and precipitates with head movements. BPPV most commonly involves the posterior semicircular canal, accounting for $95.0 \%$ of all BPPV cases. ${ }^{3}$ BPPV of the posterior semicircular canal is diagnosed by the Dix-Hallpike maneuver, which shows torsional upbeating nystagmus. The recommended management for BPPV of the posterior semicircular canal is the canalith repositioning procedure (CRP) or the modified Epley's maneuver. ${ }^{4}$ After CRP, clinicians commonly advise patients to restrict their head position or limit their activity. ${ }^{5,6}$ However, the benefit of postprocedural postural restriction remains controversial. ${ }^{4}$ Thus, the aim of this present study was to determine the efficacy of postural restriction after CRP in posterior canal BPPV patients.

\section{Material and Methods}

A single-center, prospective randomized control trial was performed at the Department of Otolaryngology, Faculty of Medicine, Prince of Songkla University from December, 2014 to May, 2018. A computer-generated block randomization was used for participant assignment into each group. This study was approved by the institutional review board of Prince of Songkla University, and written informed consent was obtained from all patients. The study adhered to the tenets of the Declaration of Helsinki and was performed in accordance with the principles of Good Clinical Practice and the Consolidated Standards of Reporting Trials statement (Figure 1). The trial was registered at the Clinical Trials Registry (NCT02475239).

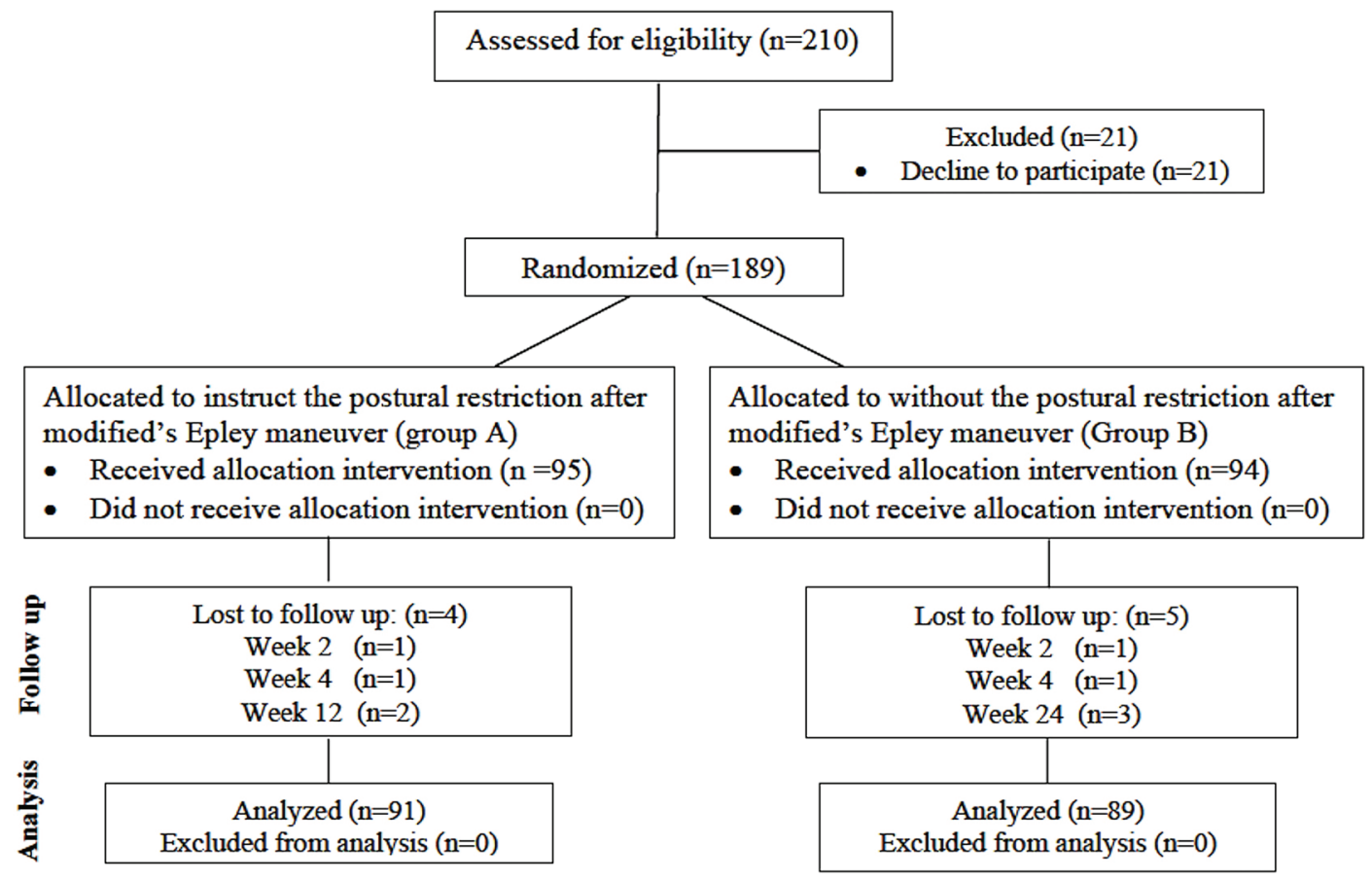

Figure 1 Consort diagram 
Two hundred and ten patients were assessed for eligibility, 189 of whom satisfied the inclusion criteria. The enrolled patients ( $>18$ years of age) had a primary BPPV of the posterior semicircular canal, which showed vertical upbeating and rotational nystagmus toward the lower ear on the Dix-Hallpike test. The exclusion criteria were: (1) secondary BPPV (the secondary BPPV was defined as patients whom had conditions associated with inner ear disorders or head trauma), (2) use of vestibular suppressants within 48 hours before treatment, (3) bilateral BPPV, and (4) neurological, musculoskeletal, or unstable cardiovascular diseases. The patients were randomized at a $1: 1$ ratio into the two treatment groups. In group $A$ (95 patients), patients were instructed to practice positional restriction after the modified Epley's maneuver. Postural restriction included wearing a soft cervical collar during the daytime, and sleeping in a semi-upright position during nighttime, for two consecutive days to avoid certain head movements. In group B (94 patients), patients received only the modified Epley's maneuver, without postural restrictions.

The allocation was performed using a sealedenvelope system. Both the clinicians and participants were unmasked to the treatment allocation. Pre-procedure data collected included: age and gender of the patient, history of ear infections or ear surgery, history of head trauma, duration of the vertigo attack, the time interval from the first vertigo attack until their outpatient department visit, and the episode (first or recurrent) and dizziness handicap inventory score $(\mathrm{DHI})$. The $\mathrm{DHI}$ score, reported by Jacobson and $\mathrm{Newman}^{7}$, evaluates the dizziness associated with physical, functional, and emotional incapacities. It contains 25 self-assessment questions, and the total score (0-100 points) is calculated by summing the ordinal scale responses.

\section{Follow-up evaluations and outcomes measures}

One hundred and eighty patients were analyzed with complete follow up. Follow-up was arranged at 1, 2 and 4 weeks after treatment. The conversion of the DixHallpike test was the primary outcome measure. The DHI score and the recurrence rate were used as secondary outcomes. We also performed telephone interviews to determine the $\mathrm{DHI}$ scores and identify patients with recurrent vertigo at 12, 24 and 48 weeks after treatment.

\section{Results}

The median age was 57 years in group $A$ and 59 years in group B. There were significantly more females affected $(75.8 \%$ in group A, $69.1 \%$ in $B)$ than males (24.2\% in group A, $30.9 \%$ in group B). The right posterior semicircular canal was the most affected side in both groups. There were no significant differences in age, gender, or affected side between the two groups, and no differences in the number of vertigo attack episodes ( $p$-value $=0.881$, chi-square test) or the number of CRPs ( $p$-value $=0.651$, Fisher's exact test). Furthermore, there were no differences in the rate of conversion of the DixHallpike test results from positive to negative at week 1 and week 2 between the two groups ( $p$-value=0.720, $p$-value $=0.701$, Table 1)

Table 1 Dix-Hallpike test results

\begin{tabular}{llll}
\hline $\begin{array}{l}\text { The results of the } \\
\text { Dix-Hallpike test }\end{array}$ & Group A & Group B & p-value \\
\hline Week 1 & $(n=95)$ & $(n=94)$ & \\
Negative & $68.4 \%$ & $66.0 \%$ & $0.720^{*}$ \\
Positive & $31.6 \%$ & $34.0 \%$ & \\
Week 2 & $(n=94)$ & $(n=93)$ & \\
Negative & $84.0 \%$ & $87.0 \%$ & $0.701^{*}$ \\
Positive & $26.0 \%$ & $23.0 \%$ & \\
\hline
\end{tabular}

${ }^{*}$ Chi-square test 
The median DHI score of the patients before treatment was 56.5 in group $A$ and 51.9 in group $B$ ( $p$-value $=0.188$, Wilcoxon Rank sum test). At 1 week after treatment the $\mathrm{DHI}$ scores of the patients in the postural restriction group and the non-restriction group were 17.9 and 28 , respectively, while at 2 weeks after treatment the $\mathrm{DHI}$ scores were 5.8 and 13.1, respectively (Figure 2). There was a significant difference in the $\mathrm{DHI}$ scores at 1 week and 2 weeks after treatment in both groups ( $p-$ value $=0.003, p=0.004$, respectively), while there were no differences at 4, 12, 24 or 48 weeks after treatment $(p-$ value $=0.080, p-$ value $=0.136, p-$ value $=0.909, p-$ value $=$ 0.712 , respectively).

The recurrence rate was defined as the number of patients presenting with another episode of vertigo following the complete resolution of the symptoms of their previous episode. A significantly higher recurrence rate was detected in group B (11.2\%) compared with group A $(3.2 \%),(p-v a l u e=0.042)$. There was no difference in the median time to complete remission between group A (10 days) and group B (14 days). However, the median time to recurrence was longer in the postural restriction group than that in the non-restriction group ( $p$-value=0.029, Table 2).

Table 2 Clinical outcome after treatment compared between group $A$ and group $B$

\begin{tabular}{llll}
\hline Clinical outcomes & $\begin{array}{l}\text { Group A } \\
(\mathbf{n}=95)\end{array}$ & $\begin{array}{l}\text { Group B } \\
(\mathbf{n}=94)\end{array}$ & p-value \\
\hline $\begin{array}{l}\text { Remission } \\
\text { Recurrence }\end{array}$ & $96.8 \%$ & $88.8 \%$ & $0.042^{*}$ \\
$\begin{array}{l}\text { Time to complete } \\
\text { remission }\end{array}$ & $3.2 \%$ & $11.2 \%$ & $0.071^{*}$ \\
$\begin{array}{l}\text { Median [days] (IQR) } \\
\text { Time to recurrence } \\
\text { (month) }\end{array}$ & $10(3,21)$ & $14(7,30)$ & \\
Median [days] (IQR) & $23(20,23)$ & $8(8,11)$ & $0.029^{*}$ \\
\hline
\end{tabular}

*Wilcoxon Ranksum test

${ }^{*}$ Fisher's exact test, IQR=interquartile range

\section{Group A Group B}

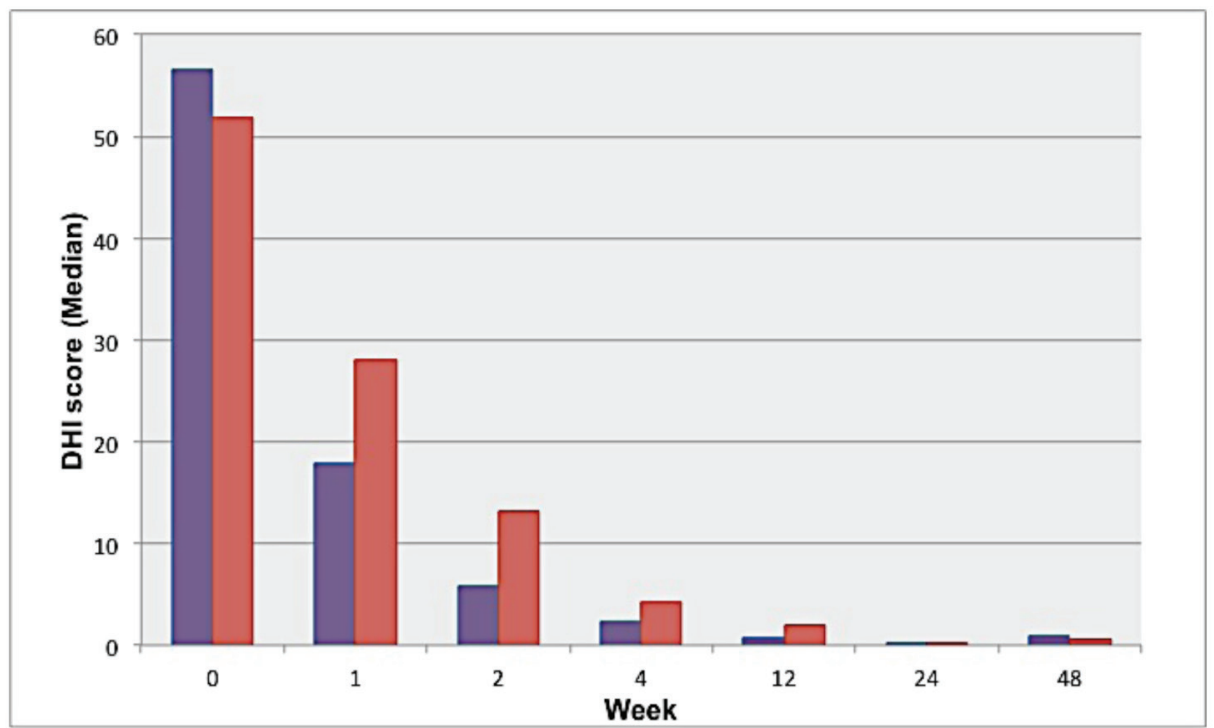

Figure 2 Dizziness handicap inventory scores before and after the repositioning maneuver in group A (postural restriction) and group B (non-postural restriction) 


\section{Discussion}

The recognized pathophysiology of BPPV involves the dislodgement and migration of otoconia from the utricular macula that enters the semicircular canals. The posterior semicircular canal usually involves otoconia, which accounts for $60.0 \%-90.0 \%$ of all cases. ${ }^{1} \mathrm{CRP}$ is the recommended treatment for BPPV, and the clinical efficacy of this maneuver is widely accepted. ${ }^{4}$ Nevertheless, BPPV patients are often requested to follow various postural restrictions to prevent the otoconia from migrating back into the semicircular canal, and to stabilize them on the utricular macula. The reported postural restrictions vary, and include use of a soft cervical collar, head elevation, limited head turning, or avoiding sleeping on the affected side. ${ }^{8}$ Further, the reported efficacy of these techniques is variable. In this present study, the compliance of the patients to the described postural restriction was considered an important variable affecting clinical outcomes. As such, we recommended use of soft cervical collars during the daytime and a sleeping pillow at nighttime, so as to help patients avoid certain head movements.

Most patients, without any instructions for postural restriction, actually avoided sleeping on the affected side or limited their head movements. However, a number of studies have demonstrated that postural restriction after the CRP does not improve the success rate of BPPV treatment, nor decrease the recurrence rate after the maneuver. ${ }^{8-12}$ Further, a more recent clinical practice guideline did not recommend the routine application of postural restrictions after the CRP. ${ }^{4}$ By contrast, Li et al. ${ }^{13}$ reported a higher recurrence rate in patients who sleep on the affected side, or who sleep in any preferred position compared with those instructed to sleep in the semi-sitting position; and refrain from sleeping on the affected side. Shim et al. ${ }^{14}$ also reported that the preferred head-lying side at the beginning of sleep correlated with the affected side by BPPV. Additionally, Cakir et al. ${ }^{5,15}$ found a significant difference in the number of maneuvers required between non-postural restriction and postural restriction treatment groups. Similarly, Hunt et al. ${ }^{16}$ reported that the addition of postural restrictions was associated with a number needed to treat 10 maneuvers, and that postural restrictions did not expose the majority of patients to any risks of harm or discomfort.

BPPV patients suffer from a limited ability to perform physical activities along with reduced emotional and social functioning, which can cause difficulties in their daily life as well as reducing their quality of life. ${ }^{17}$ Using the $\mathrm{DHI}$ questionnaire, we found that the postural restriction group experienced fewer handicaps than those in the nonpostural restriction group; suggesting that postural restriction may be beneficial if the symptoms conformed to high $\mathrm{DHI}$ scores. Residual dizziness is a common complaint of patients after successful BPPV treatments and this may be the cause of the remaining high $\mathrm{DHI}$ scores. It may be hypothesized that the dispersed otoliths in the semicircular canal or canal reentry may be an explanation of residual dizziness, and it is insufficient to elicit noticeable nystagmus with the naked eye on the Dix Hallpike test. Postural restriction may help to stabilized otoliths within the utricle and prevent otoliths migration.

Overall, these data suggest that post-procedural postural restriction should be routinely discussed with BPPV patients, and be considered a potential treatment modality for BPPV.

One limitation of this present study is the objective test, such as videonystagmography, which was not used in confirming nystgamus; this might have compromised the treatment results. Second, because of some technical limitations, we did not test serum 25-hydroxyvitamin D, which has the etiology of recurrent BPPV and is possibly associated with low serum vitamin D levels. 


\section{Conclusion}

The addition of postural restriction after the modified Epley's maneuver in BPPV patients can improve both $\mathrm{DHI}$ scores and vertigo symptoms and reduce the recurrence rate, compared with the application of the modified Epley's maneuver alone.

\section{Funding sources}

We thank the Faculty of Medicine, Prince of Songkla University for funding support.

\section{Conflict of interest}

The authors declare that they have no conflicts of interest. No author has any proprietary interest in any of the products or ideas mentioned in this article.

\section{References}

1. Kim JS, Zee DS. Benign paroxysmal positional vertigo. N Engl J Med 2014;370:1138-47.

2. von Brevern M, Radtke A, Lezius F, Feldmann M, Ziese $T$, Lempert $\mathrm{T}$, et al. Epidemiology of benign paroxysmal positional vertigo: a population based study. J Neurol Neurosurg Psychiatry 2007;78:710-15.

3. Parnes LS, Agrawal SK, Atlas J. Diagnosis and management of benign paroxysmal positional vertigo (BPPV). CMAJ 2003; 169:681-93.

4. Bhattacharyya N, Gubbels SP, Schwartz SR, Edlow JA, El-Kashlan $\mathrm{H}$, Fife $\mathrm{T}$, et al. Clinical practice guideline: benign paroxysmal positional vertigo (update). Otolaryngol Head Neck Surg 2017;156:S1-47.

5. Epley JM. The canalith repositioning procedure: for treatment of benign paroxysmal positional vertigo. Otolaryngol Head Neck Surg 1992;107:399-404.

6. Cakir BO, Ercan I, Cakir ZA, Turgut S. Efficacy of postural restriction in treating benign paroxysmal positional vertigo. Arch Otolaryngol Head Neck Surg 2006;132:501-5.
7. Jacobson GP, Newman CW. The development of the Dizziness Handicap Inventory. Arch Otolaryngol Head Neck Surg 1990; 116:424-7.

8. Devaiah AK1, Andreoli S. Postmaneuver restrictions in benign paroxysmal positional vertigo: an individual patient data metaanalysis. Otolaryngol Head Neck Surg 2010;142:155-9.

9. Balikci HH, Ozbay I. Effects of postural restriction after modified Epley maneuver on recurrence of benign paroxysmal positional vertigo. Auris Nasus Larynx 2014;41:428-31.

10. Moon SJ, Bae SH, Kim HD, Kim JH, Cho YB. The effect of postural restrictions in the treatment of benign paroxysmal positional vertigo. Eur Arch Otorhinolaryngol 2005;262:408-11.

11. Casqueiro JC, Ayala A, Monedero G. No More Postural Restrictions in Posterior Canal Benign Paroxysmal Positional Vertigo. Otol Neurotol 2008;29:706-9.

12. Mostafa BE, Youssef TA, Hamad AS. The necessity of postmaneuver postural restriction in treating benign paroxysmal positional vertigo: a meta-analytic study. Eur Arch Otorhinolaryngol 2013;270:849-52.

13. Li S, Tian L, Han Z, Wang J. Impact of postmaneuver sleep position on recurrence of benign paroxysmal positional vertigo. PLoS One 2013;8. doi: 10.1371/journal.pone.0083566.

14. Shim DB, Kim JH, Park KC, Song MH, Park HJ. Correlation between the head-lying side during sleep and the affected side by benign paroxysmal positional vertigo involving the posterior or horizontal semicircular canal. Laryngoscope 2012; 122:873-6.

15. Cakir BO, Ercan I, Cakir ZA, Civelek S, Turgut S. Relationship between the affected ear in benign paroxysmal positional vertigo and habitual head-lying side during bedrest. J Laryngol Otol 2006;120:534-6.

16. Hunt WT, Zimmermann EF, Hilton MP. Modifications of the Epley (canalith repositioning) manoeuvre for posterior canal benign paroxysmal positional vertigo (BPPV). Cochrane Database Syst Rev 2012;4:CD008675.

17. Lopez-Escamez JA, Gamiz MJ, Fernandez-Perez A, GomezFinana M. Long-term outcome and health-related quality of life in benign paroxysmal positional vertigo. Eur Arch Otorhinolaryngol 2005;262:507-11. 